\title{
Introducción al periodismo de investigación contemporáneo en la prensa estadounidense
}

\section{Francisco M. Casal}

Doctor en Periodismo

\section{Resumen:}

Pese a que el Periodismo de Investigación contemporáneo en los EE.UU. hunde sus raíces en una tradición periodística cuyos albores se vislumbraron ya en el siglo XVIII, no ha sido hasta la segunda mitad del siglo xx cuando los profesionales lograron establecer una práctica sistemática de la especialidad gracias al Proyecto Arizona y a la fundación y desarrollo del Investigative Reporters and Editors Inc. Hoy supone la máxima excelencia profesional del periodismo norteamericano.

Palabras clave:

'Muckrakers' - periodismo de investigación - historia del periodismo - periodismo asistido por ordenador

Abstract:

Despite the fact that Investigative reporting roots can be found back in the late $18^{\text {th }}$ Century, it was during the last decades of the $20^{\text {th }}$ one when professional journalists achieved a systematic practice of the craft due to the success of the Arizona Project and the founding and development of Investigative Reporters and Editors Inc. Today Investigative Reporting means the highest level of excellence in U.S. journalism.

Key words:

Muckraking - investigative reporting - journalism history - computer - assisted reporting.

\section{Introducción}

Aunque la mayoría de los historiadores del periodismo coinciden en asociar el nacimiento del Periodismo de Investigación con la aparición del movimiento "muckraker", los albores de la especialidad pueden ser situados con cierta claridad documental en 1721 cuando James Franklin -el hermano de Benjamín- 
criticó en The New England Courant los planes del gobierno en Boston para vacunar a la población contra la viruela (Aucoin, 1997a). Otros historiadores del periodismo estadounidense mencionan los trabajos de pioneros de la investigación periodística involucrados en la denuncia de asuntos que las autoridades de la colonia preferían que no se hicieran públicas (cfr. Protess et al., 1991:29 y ss.). Posteriormente, en la década de 1880, Henry Demarest Lloyd publicaría una serie de artículos exponiendo la corrupción dominante en la política y en los negocios de los EE.UU. del momento. Concretamente, la serie incluía "The Story of a Great Monopoly" (1881) y "The Political Economy of Seventy-Three Million Dollars" (1882), ambas publicadas en el Atlantic Monthly, junto con "Making Bread Dear" (1883) y "Lords of Industry" (1884), difundidas estas a través de la North American Review. Estos cuatro artículos causaron semejante revuelo que Lloyd fue descrito desde entonces como el primer periodista de investigación estadounidense. (Aucoin, 1997a)

Otro de los nombres destacados del albor de la especialidad es el de Nellie Bly, quien a sus dieciocho años trabajaba para el Pittsburg Dispatch. El estilo periodístico de Bly estaba marcado por las historias de primera mano sobre las vidas de la gente de la calle; solía conseguir sus reportajes a base de aventuras que ella misma vivía de forma encubierta, inventando personalidades e identidades ficticias que le permitían contar "desde dentro" una situación. En 1887 Bly fue contratada por Joseph Pulitzer para escribir en el New York World y en los siguientes años se dedicó en exclusiva a escribir sobre la pobreza, las condiciones de vida y de trabajo de los neoyorquinos, lo que a menudo le obligaba a vivir directamente las condiciones de vida que pretendía denunciar, llegando incluso a fingir enfermedades para poder acceder al manicomio de Blackwell's Island, donde descubrió cómo los pacientes eran alimentados con comida infestada de sabandijas y sufrían todo tipo de abusos por parte del personal que les atendía. Descubrió además que algunos pacientes no estaban psicológicamente enfermos pero sí sufrían enfermedades físicas o habían sido recluidos allí a la fuerza por sus familiares. Las duras críticas vertidas en el periódico por Bly sobre las condiciones de aquel manicomio obligaron a las autoridades a efectuar reformas para solventar aquellas situaciones (Kroeger, 1994).

Otro temprano ejemplo de Periodismo de Investigación fue el trabajo de Jacob A. Riis para el Scribner's Magazine en 1899 con una serie de artículos titulados "How The Other Half Lives". En diciembre de ese año Benjamín Flower fundaba la revista The Arena, especializada en este tipo de periodismo. Durante sus primeros años de vida Flower publicó un gran número de artículos sobre pobreza, explotación laboral, viviendas inhabitables, desempleo y trabajo infantil.

Pero el Periodismo de Investigación se convirtió en un movimiento en sí mismo cuando en 1902 publicaciones como McClure's Magazine y Everybody's Magazine se unieron al Arena en la lucha por las reformas sociales. Fueron publicaciones que alcanzaron un tremendo éxito popular inmediatamente seguido por otras como Cosmopolitan y el Saturday Evening Post que pronto publicaron artículos denunciando corrupción política y empresarial. En 1906 las ventas combinadas de las diez revistas en las que 
se concentraba la producción de Periodismo de Investigación del momento alcanzaban una difusión de tres millones de ejemplares.

Poco tiempo después, muchos de los periodistas que escribían en ellas cimentando la especialidad, usaron los materiales que habían publicado para convertirlos en novelas y libros de no ficción. Charles Edward Russell llegó a vender treinta mil ejemplares de cada una de las muchas novelas que publicó basadas en investigaciones periodísticas. Upton Sinclaire fue, sin embargo, el más exitoso novelista de este grupo de pioneros. Sus novelas The Jungle y The Brass Check fueron best-sellers que superaron los cien mil ejemplares a principios del siglo xx. La nómina de escritores y editores asociados con la irrupción del Periodismo de Investigación entre 1900 y 1914 incluía también a Frank Norris, Ida Tarbell, Lincoln Steffens, David Graham Phillips, C.P. Connolly, Benjamín Hampton, Thomas Lawson, Alfred Henry Lewis y Ray Stannard Baker, entre otros.

La repercusión de aquel movimiento -los famosos "Muckrakers", rastreadores de basura- produjo que el entonces presidente Theodore Roosevelt tomase iniciativas legislativas para paliar algunos de los problemas expuestos por aquellos periodistas, lo que incluía persuadir al Congreso para aprobar reformas como la Pure Food and Drugs Act o la Meat Inspection Act, ambas de 1906. La aparición y desarrollo, así como el declive de la era de los Muckrakers merecería por su significación e importancia en la historia de la especialidad, ser tratado en una monografía específica, y eso es algo que excede la intención de este artículo.

Tras la desaparición de tan intensa producción investigadora pocos años después, comienza un largo período de relativo vacío en lo que a la existencia del Periodismo de Investigación se refiere que durará, salvo algún trabajo aislado, hasta entrada la sexta década del siglo xx. Expertos historiadores de la especialidad como James Aucoin, de la University of South Alabama, rehúsan afirmar que la especialidad hubiese desaparecido por completo: "Efectivamente existe un agujero en el tiempo, lo que no significa necesariamente una total ausencia", afirma Aucoin (comunicación personal, septiembre de 2003), "el problema es que la mayoría de las investigaciones de esa época eran muy locales, se exponían corrupciones de los policías locales, ese tipo de cosas". Una historia de alcance nacional que mantuvo viva la especialidad fue, en 1924, el escándalo de Teapot Dome, en el que el gobierno federal vendió reservas de petróleo a las petroleras a muy bajo precio como pago de sobornos.

En esencia, la especialidad continuó viva en los cincuenta años siguientes a la época dorada de principios de siglo gracias a pequeñas revistas como Nation o a los libros de no ficción con investigaciones periodísticas. Quizá el mejor referente durante ese agujero temporal al que Aucoin hace referencia lo constituya lo que hoy denominaríamos una columna de opinión. Se trataba de una columna sindicada (de agencia) realizada a dúo en los años treinta por Drew Pearson y Robert S. Allen titulada Washington Merry-Go Round. El valor de aquella columna radicaba en que ofrecía investigaciones con datos noticiosos antes de que otros periodistas tuviesen acceso a ellos. 
Otro ejemplo que demuestra que el Periodismo de Investigación continuó vivo durante las poco estudiadas décadas de los 40 y 50 es el reportaje que Edgard Morrow realizó en 1954 para la cadena CBS dentro del programa "See It Now" en el que denunciaba y documentaba minuciosamente las "inconsistencias", "medias verdades" y "distorsiones" del tristemente célebre senador McCarthy. El reportaje produjo una intensa reacción popular e institucional que desembocó en la caída en desgracia del senador ultraderechista. Posteriormente Morrow realizaría otra investigación sobre las condiciones de trabajo de los inmigrantes en las explotaciones agrícolas norteamericanas (Protess et al., 1991). Ejemplos como los mencionados demuestran que la especialidad continuaba viva en los 50 aunque, como coinciden Protess y su equipo (1991), Aucoin (comunicación personal, septiembre de 2003) y Dader (1997:34) el espíritu dominante de la época del "periodismo objetivista" haya hecho que no se haya recordado lo suficiente ${ }^{1}$.

Fue durante los turbulentos años 60 cuando la especialidad emergió definitivamente como una práctica descollante en el periodismo norteamericano. De hecho, a mitad de los 70 había ya madurado significativamente aunque no podía aún entenderse fuera de las empresas periodísticas que publicaban los resultados de las investigaciones. Los proyectos individuales de aquel momento como los de Donald Barlett y James Steele sobre el sistema de justicia penal de Philadelphia para el Inquirer y la investigación de la corrupción policial en Chicago hecha por el equipo del Chicago Tribune empujaron hacia delante tanto en lo que a las técnicas de investigación como a los estándares de calidad se refiere ${ }^{2}$. En cualquier caso, pese a estas nuevas espoletas, la especialidad continuó sin ser tenida demasiado en cuenta y adolecía de una estructura profesional concreta, según afirma Aucoin (1995b). Incluso entre los periodistas, esa incertidumbre permanecía en el sentido de dilucidar si se trataba de algo diferente de otras formas conocidas de periodismo o si era una etiqueta innecesaria puesta sobre algo que siempre había sido buen y sólido periodismo.

Pero probablemente más que ningún otro factor, fue sin duda la investigación periodística del escándalo Watergate, lo que contribuyó a recobrar la moda de la investigación periodística y fraguó los cimientos de una especialidad periodística diferenciada. La proliferación de imitadores de Bernstein y Woodward se vio por primera vez acompañada de reformas internas en las redacciones para crear "uni-

\footnotetext{
${ }^{1}$ Para encontrar otros ejemplos de Periodismo de Investigación de la época, puede consultarse Serrin, J. / Serrin, W. (2002) Muckraking! The Journalism That Changed America. New York. New Press., donde se recopila y comenta uno a uno ciento cincuenta trabajos de investigación periodística norteamericanos desde los muckrakers hasta el presente.

${ }^{2}$ Un análisis sobre el trabajo de Barlett y Steele puede revisarse en Meyer, P. (1979:336-386). Asimismo, para una mayor profundización, véase Weinberg, S. (1992:111-112). En cuanto a las series del Chicago Tribune, publicadas en noviembre de 1973, puede revisarse la reimpresión de las mismas bajo el título "Police Brutality" y la revisión del caso que hace Dygert (1976: 129-130).
} 
dades de Periodismo de Investigación" y de la aparición de libros académicos o profesionales que pretendían fijar el "canon" de la supuestamente nueva especialidad ${ }^{3}$.

Durante los años 70 se publicaron libros sobre la especialidad, tanto de texto como comerciales pero por lo general no tenían una calidad suficiente como para ser considerados propuestas sistemáticas, aunque eran los más recurridos a la hora de definir y hablar sobre el tema.

Usando la terminología propuesta una década después por el filósofo Alasdair MacIntyre, los periodistas de mitad de los 70 se preguntaban si el Periodismo de Investigación era una práctica social distinta. Es una pregunta que permaneció en el aire incluso hasta los años 90 y que puede ahora ser contestada con mayor precisión gracias al trabajo de MacIntyre aunque en él no aborde el periodismo directamente sino cómo las prácticas sociales pueden ser analizadas usando la filosofía, la filosofía moral, la historia y la sociología (1981).

MacIntyre define la práctica social, siguiendo el criterio dominante en la teoría sociológica contemporánea, como una actividad humana coherente, compleja y cooperativa en un ambiente social. Según él los miembros de una práctica obtienen beneficios específicos de esa práctica a través de actividades que persiguen un estándar de excelencia. Argumenta que la práctica social se desarrolla y se sostiene a través de los esfuerzos de sus practicantes llegando a conseguir el objetivo y ampliando los estándares de excelencia (Ibíd.).

Antes de la fundación del Investigative Reporters and Editors (IRE) a mediados de la década de los 70, el Periodismo de Investigación, en opinión de Aucoin, no alcanzaba los requisitos para ser una práctica social determinada según el criterio de MacIntyre. La fundación del IRE en 1975 estableció en sí misma las bases para el desarrollo del Periodismo de Investigación como actividad social específica. Hasta mediados los 70, el Periodismo de Investigación era una tarea solitaria e individualista. Su escala se puede observar en biografías, autobiografías y otros tipos de investigaciones publicadas a mediados de aquella década. Jack Anderson, por ejemplo, publicó su autobiografía en el 73 bajo el título de The Anderson Papers, enfatizando su rol de investigador solitario (Anderson, 1973). Joe Eszterhas realizó un perfil de Seymour M. Hersh para Rolling Stone y lo tituló "el reportero más duro de América”, retratando, como Anderson, al periodista de investigación como un bicho solitario (Eszterhas, 1977). Y John C. Behrens publicó un libro sobre los periodistas de investigación como hombres solitarios, "The Typewriter Guerrillas: Closeups of 20 Top Investigative Reporters" (1977). En estos y otros trabajos había muy pocas referencias al Periodismo de Investigación como una comunidad de practicantes con su conjunto de

\footnotetext{
${ }^{3}$ Para una referencia pormenorizada del caso Watergate, entre la ingente literatura editada sobre el tema, puede consultarse la obra de Buschel et al. (1973) The Watergate Files. New York. Flash Books, dada la estructuración de la obra en secuencias cronológicas, detalles de quién fue quién en el caso y un completo análisis sobre las implicaciones políticas y constitucionales del escándalo.
} 
estándares y destrezas. El periodista individual era ensalzado como un simple héroe solitario disparando a los malos. Ese mito es recogido por Benjamín y Anderson en un texto sobre la especialidad:

Todo el mundo sabe lo que es un periodista de investigación. Es ese personaje con un cigarrillo colgado de los labios, el rostro pálido y el abrigo con botones que entra y sale de las cabinas telefónicas, habla con la esquina de su boca e ignora a los demás periodistas. Nunca ha tenido que aprender su oficio, nació para él. Salió del vientre de su madre con una agenda colgando del cuello y el certificado de nacimiento de su auténtico padre. Tiene una interminable lista de contactos, su trabajo consiste básicamente en llamarles y decirles "dame algo"... Aparece en la redacción sólo cada dos o tres meses para dejar sus textos en la mesa de los atónitos editores, murmurar unas pocas palabras y desaparecer de nuevo en la noche. (Benjaminson y Anderson, 1990)

Pero esta imagen no es suficiente para argumentar que los periodistas de investigación no existen (o existen solo bajo ese estereotipo cinematográfico). De hecho, Francke ha documentado el uso de equipos de investigación a finales del siglo XIX por parte del editor del Leslie's Weekly, Frank Leslie, así como por el New York Times y el St. Louis Post-Dispatch (Francke, 1988). Pero estos equipos fueron individualistas: cada uno trabajaba para un único periódico o medio y raramente se comunicaban con los compañeros que no fueran de su medio respecto de ideas, fuentes, técnicas o cualquier otro asunto relacionado con la tarea de rastrear corrupciones o irregularidades.

Sí había alguna cooperación cuando dos o mas periodistas resultaban estar trabajando en la misma historia. Jack Newfield, del Village Voice, por ejemplo contó a un entrevistador a mediados de los $70 \mathrm{su}$ cooperación con John Hess, del New York Times, y Steve Barman, de la WNEW-TV, a lo largo de una investigación sobre el responsable de las casas de acogida de Nueva York, Bernard Bergman, en 1974. Los tres periodistas no trabajaron juntos, según Newfield, pero sí intercambiaron ideas (Aucoin, 1995). Y dado que estaban apareciendo historias sobre la misma persona en tres medios locales diferentes al mismo tiempo, los hallazgos de fraude sanitario por parte de Bergman no pudieron ignorar la estructura de poder.

Ese aislamiento y la confusión sobre si la especialidad existía se produjo inclusive pese a los esfuerzos de algunos académicos del periodismo por impartirla. Algunas facultades incluían cursos de Periodismo de Investigación y había organizaciones profesionales que ofrecían seminarios sobre el tema (Williams, 1978; McWilliams, 1973). Por ejemplo, el American Press Institute (API) ofrecía desde 1961 seminarios periódicos sobre Periodismo de Investigación (Christianson, 1972) .

Pero, pese a todo, el Periodismo de Investigación continuaba definido como un área ("beat” en el argot profesional norteamericano), no como una práctica claramente diferenciada dentro del periodismo.

${ }^{4}$ El American Press Institute realizó seminarios en 1961, 1963, 1969, 1971 (dos), 1973 y 1974, según se menciona en los archivos de Paul Williams, IRE Resource Center, University of Missouri School of Journalism. 
La celebración de un congreso sobre la cobertura mediática de asuntos públicos en 1973 fue conmocionada por las investigaciones del escándalo Watergate y los oradores comenzaron a afirmar que el Periodismo de Investigación era "la llamada más alta" de un reportero (Freivogel, 1973). Los participantes en aquel congreso vieron al Periodismo de Investigación como una extensión del periodismo "de asuntos públicos", como algo que los periodistas hacían al cubrir información sobre los ejecutivos públicos, el medioambiente o la educación (Ibíd.). Dos años más tarde se celebró otro congreso específicamente dedicado a valorar "las lecciones del Watergate" y Joe Heaney, del Boston Herald-American, sugirió que todos los periodistas debían ser reconocidos como periodistas de investigación (Kirtz, 1975); Robert Maynard, editorialista del Washington Post urgió a sus colegas a "no dejarse encasillar al pensar en términos de periodistas de investigación y el resto de nosotros. Me preocupa que el término se mistifique. Se supone que es lo que somos todos nosotros durante todo el tiempo", afirmó. (Ibíd.)

En la cima del resurgir del Periodismo de Investigación, tras el Watergate, incluso cuando los estudiantes se lanzaban sobre el trabajo de los muckrakers de principios de siglo para rescatar las raíces de la especialidad y probar así una continuidad histórica de la misma (Stein, 1979; Miraldi, 1990), e incluso mientras los escritores populares declaraban héroes a los periodista de investigación, muchos otros periodistas seguían sin tener clara la distinción entre un periodistas de investigación y el resto de la profesión. Escondida tras una visión mítica del periodista investigador como un individualista y un héroe popular residía un prejuicio institucional. Los periodistas de investigación eran vistos como un producto de un medio compuesto por periodistas individualistas, no como el producto de esos mismos individuos. Por ejemplo, el tema principal de un seminario sobre periodismo y desvelamiento (en el sentido investigador anglosajón del término 'exposé') en 1973 era que la gestión era la clave del Periodismo de Investigación (Watson, 1973). Los participantes, incluido el responsable de equipos de investigación de Los Angeles Times y del Riverside Press Enterprise, hicieron hincapié en que todos los periódicos, independientemente de su tamaño, podían hacer investigaciones si sus responsables se lo proponían y asignaban a ello personal. No se necesitaban conocimientos, técnicas o entrenamiento a su juicio. Si todos los periodistas eran investigadores, entonces los periodistas de investigación no eran nada único; no era un género desde su punto de vista, sino una extensión de rutina de la cobertura de asuntos públicos.

La tensión entre la predominancia de una institución y la autonomía del periodista individual que ha sido discutida por Boylan y otros se aplica de igual forma a la relación entre la gestión de un medio y los periodistas de investigación (1986). Sin una comunidad de periodistas investigadores fuera de la estructura institucional de las empresas informativas el azar de la especialidad quedaba determinado por las instituciones, las empresas, un factor que MacIntyre definió como en detrimento del desarrollo de una práctica social. Sin un enfoque en la práctica, sin el reconocimiento de que el Periodismo de Investigación era, en efecto, una especialidad que requería especialización y producía reportajes y series 
de un carácter diferente al de otro periodismo, no podía darse un desarrollo sostenido y sistemático de la especialidad.

Quizá el mejor indicador de la falta de una comunidad entre los periodistas de investigación a mediados de los setenta fuesen los fundadores del IRE. La frustración del sentimiento de aislamiento y la percepción de que una organización nacional podría realzar la práctica de la especialidad fueron las claves que motivaron el nacimiento de la organización. En 1973, Harley R. Bierce y Myrta J. Pulliam, que lideraban el equipo investigador del Indianapolis Star junto con Richard E. Cady y William Anderson, comenzaron la primera investigación en equipo para el Star, sobre el departamento de policía de la ciudad, que duraría seis meses. Descubrieron sobornos, extorsión y robos por parte de los policías en Indianapolis y consiguieron, entre otros premios, un Pulitzer (Dygert, 1976). En investigaciones posteriores trataron de exponer la corrupción policial a nivel nacional. Este gran proyecto sería la germinación de una semilla que daría lugar a una organización nacional de periodistas de investigación, semilla que había sido plantada por el periodista del Chicago Tribune Ron Koziol con la ayuda del académico Paul Williams, anterior ganador de un Pulitzer como editor de investigación ${ }^{5}$.

Mientras trabajaban en la investigación de la corrupción policial a escala nacional, Bierce y Pulliam experimentaron en carne propia las limitaciones de conocimientos y técnicas con las que el Periodismo de Investigación se topaba al iniciar una investigación. La falta de contactos entre los periodistas alrededor del país supuso un detrimento serio y ambos supusieron que una red de periodistas dispuestos a colaborar entre todos simplificaría la realización de los reportajes que no estuviesen solo enfocados al ámbito local (Editor \& Publisher, 8-III-1975) ${ }^{6}$. En consecuencia, comenzaron a debatir la utilidad de una organización de servicio nacional para periodistas de investigación.

A la primera reunión se invitó a editores, reporteros, educadores, y empresarios del ramo, así como a otros que en el pasado habían hecho Periodismo de Investigación y se propuso y aprobó el nombre de Investigative Reporters and Editors?

Desde el principio, los organizadores del IRE expresaron su preocupación por definir al Periodismo de Investigación de la forma más amplia posible. Adoptando la definición realizada por Robert Green, del Newsday, definieron un trabajo periodístico de investigación como aquel resultado de una iniciativa

\footnotetext{
${ }^{5}$ Según se recoge en "IRE-Background", folleto informativo repartido durante el primer congreso nacional del IRE en junio de 1976. Citado en Aucoin (1995).

${ }^{6}$ Una mayor documentación sobre la fundación de esta red primigenia puede consultarse en Harley R Bierce a J. Montgomery Curtis, 6 de febrero de 1975, carta titulada "organizacional setter", Archivos del IRE, IRE Resource Center, University of MissouriColumbia School of Journalism.

7 Para una descripción pormenorizada de la génesis de la organización, Cfr. Casal, F. (2005) El Periodismo de Investigación Asistido Por Ordenador y de Precisión aplicado a la prensa local y regional: el contraste entre Estados Unidos y un modelo de futuro para Galicia. Tesis doctoral. Universidad Complutense de Madrid.
} 
y de un trabajo personal del periodista (por ejemplo, aquel que no fuese resultado de una investigación realizada por estamentos externos como la policía o el poder judicial); que versase sobre un tema de relevancia e importancia para la audiencia; y que hiciese pública información que algo o alguien desease mantener en secreto ${ }^{8}$. La definición fue controvertida, particularmente en su énfasis sobre revelación de secretos, pero representaba la primera definición oficial y generalmente aceptada sobre la especialidad. Desde aquel 1976, el IRE se estableció en cooperación con la facultad de periodismo de la Universidad de Missouri-Columbia, se dotó de personal laboral, fundó el IRE Journal como órgano de comunicación entres sus miembros, estableció congresos anuales nacionales y regionales y desarrolló un programa de premios anuales con criterios que todavía hoy representan el mejor estándar del Periodismo de Investigación norteamericano.

Tras definir a la especialidad, el IRE jugó el papel clave en la realización del Proyecto Arizona, durante el que miembros de la organización investigaron la corrupción existente en el estado ${ }^{9}$. El Proyecto, que constaba de una serie de reportajes publicados entre noviembre de 1976 y marzo de 1977 en diferentes diarios, fue ideado en respuesta al asesinato del reportero Don Bowles, y fue reconocido a escala nacional como paradigma de calidad investigativa. Sin embargo, supuso un sinfín de problemas, tanto legales y financieros como de organización interna, que casi logran dar al traste con la organización recién nacida. Era, sin embargo, parte integral del intento del IRE de establecer una comunidad sólida de periodistas de investigación.

Antes del nacimiento del IRE no existía ningún archivo donde consultar las investigaciones periodísticas publicadas hasta el momento. En esencia, la organización de Missouri ha documentado la historia del Periodismo de Investigación en Estados Unidos desde sus inicios, a través del mantenimiento de una "morgue" de investigaciones con ejemplos del Periodismo de Investigación realizado en dicho país. Esta biblioteca, junto con los seminarios académicos realizados durante y entre sus congresos anuales y regionales, y sus publicaciones, aporta a los periodistas de investigación un diálogo continuo con el pasado de su práctica.

Precisamente ese potencial de fortalecimiento y progreso que la actividad conjunta en torno a una práctica es capaz de desarrollar dentro de una comunidad coordinada, constituye sin duda uno de los factores de la rápida evolución en el periodismo estadounidense de investigación hacia nuevas estrategias

\footnotetext{
${ }^{8}$ Carta de John Ullman a los miembros ejecutivos del IRE, 30 de mayo de 1979. En los Archivos del IRE bajo el título de "1979 Original awards criteria".

${ }^{9}$ Véase "The Arizona Project: Reprint of a 1977 series", IRE, 1977. Para una revisión del proyecto Arizona, puede consultarse Senia, A. (1978): “The Arizona Project. A Year Later”. The Quill, julio-agosto 1978 pp. 10-28; Mollenhoff, C. (1981) Investigative Reporting: from Courthouse to White House. New York. MacMillan; o la obra de Wendland, M. (1988): The Arizona Project : how a team of investigative reporters got revenge on deadline. Kansas City. Sheed Andrews and McMeel, así como la sección dedicada al proyecto accesible en la dirección www.ire.org/history/arizona.html
} 
y técnicas de investigación que enlazan con el llamado "Periodismo de Precisión” y "Asistido por Ordenador", que lleva aparejada una investigación periodística sistemática, en contraposición a la interpretación y destilación de información ya conocida de antemano que caracterizaba a los investigadores de principios del siglo $\mathrm{xx}$.

Ya en la década de los años 60 se había producido un intento de desarrollar una metodología sistemática del Periodismo de Investigación cuando Virginia J. Montgomery Curtis, del American Press Institute (API), Ben Reese, ex-director del St. Louis Post-Dispatch, John Seigenthaler, del Nashville Tennessean y Clark Mollenhoff, de Cowles Papers detallaron métodos y guías de investigación periodística que pudieran ser aplicadas para investigar al gobierno, instituciones privadas o agencias públicas. En 1976, Mollenhoff describía ese sistema como un "análisis de la historia de la institución, sus objetivos, y un estudio de cómo esos objetivos avanzan desde un punto de partida de posibles conflictos de intereses y la administración de sus leyes y reglamentos legales. Tiene una completa guía para el periodista investigador, de forma que no olvide cualquier área de potencial irregularidad o corrupción"10.

Aquel sistema marcó el estándar para abarcar la cobertura y profundidad en el periodismo, y se convirtió en la base de una serie de seminarios sobre la especialidad patrocinados por el API desde 1968. Dicho estándar fue desarrollado posteriormente por Paul Williams en su clásico de 1978, Investigative Reporting and Editing.

Tras entrevistar a 99 periodistas y editores de investigación a mediados de los 70, Williams produjo uno de los primeros modelos de metodología para el Periodismo de Investigación partiendo de la premisa de que se trataba de un proceso intelectual. Los proyectos de investigación, consciente o inconscientemente, se desarrollan a través de varios pasos que van desde la concepción hasta la publicación final. En el gran número de pasos intermedios debían efectuarse evaluaciones del trabajo. David Protess encabezaría más de una década después un equipo de investigadores para alcanzar la misma conclusión de que el Periodismo de Investigación conlleva "procesos altamente distintivos" que ocupan un tiempo considerable, e incluyen la aplicación de normas explícitas e implícitas, además de generalizaciones desde hechos específicos hasta grandes asuntos sociales. A través del proceso, los periodistas y editores reevaluarían constantemente la concepción del trabajo y, aunque se podía argumentar que gran parte de ese proceso también se realiza durante el periodismo convencional de cierre diario, el énfasis sobre la investigación original, la compartimentación de la historia como una mirada exhaustiva de un problema público y el retraso de las entrevistas clave hasta que la mayor parte de la investigación estuviese completada, separaban al Periodismo de Investigación del periodismo convencional.

El profesor James Aucoin, en su "The Re-emergence of American Investigative Journalism (19601975)"(1995b), argumenta que la reaparición de la especialidad a partir de los años 60 se debió a factores

\footnotetext{
${ }^{10}$ Citado en Aucoin (1993a:13).
} 
sociales y culturales como los movimientos por los derechos civiles, la guerra de Vietnam o el hecho de que los periodistas alcanzasen un status social más elevado que en las décadas anteriores. En su opinión se habría debido también a desarrollos en las leyes de prensa y la Primera Enmienda emanados de casos de la Corte Suprema y del trabajo de los teóricos del Derecho. Asimismo menciona la aparición de la prensa alternativa y underground contemporánea, los avances en la tecnología aplicada a la producción de periódicos como las grabadoras y los ordenadores y a la legislación sobre libertad de información.

En el resurgimiento y aun la espectacular puesta de moda que el Periodismo de Investigación -genuino o de imitación bajo los más variopintos sucedáneos-, tuvo sin duda una importancia capital la publicación y el eco del caso Watergate, entre 1972 y 1973. Pero dicho trabajo podría haberse quedado en un episodio aislado, de no haber sido por otros esfuerzos más colectivos y sostenidos que, beneficiándose sin duda del impacto logrado por Bernstein y Woodward, contribuyeron a sistematizar el Periodismo de Investigación y a hacerlo reconocible como una perspectiva diferenciada y utilizable por cualquier periodista o equipo dispuesto a someterse a su disciplina. En 1975 la especialidad evolucionó hacia una práctica madura y viable que era parte, aunque con características distintas, del periodismo tradicional. Durante las décadas de los 60 y 70, la práctica de la investigación periodística desarrolló conocimientos técnicos para la investigación de asuntos públicos y se establecieron unos estándares de excelencia. En ese contexto nacía el Investigative Reporters and Editors y el desarrollo del 'Proyecto Arizona' resultó ser su inmejorable tarjeta de presentación, además de un hito en lo que se refiere a la práctica de la especialidad. Todavía hoy no reconocido como tal en comparación con otras investigaciones que han pasado a los anales del gran periodismo, el Proyecto Arizona se convirtió en el disparo de salida de una tendencia que duraría hasta la actualidad.

Don Bolles, con 47 años, se había ganado su reputación como periodista a base de publicar las irregularidades políticas y las actividades del crimen organizado en Arizona a lo largo de los catorce años que llevaba trabajando para el Arizona Republic. Este tipo de revelaciones a lo largo de aquellos años le habían hecho obtener varias candidaturas para el Pulitzer, poseedor de una sólida reputación entre la comunidad de periodistas de investigación norteamericanos, y finalmente le costaron la vida en un atentado con bomba al encender el motor de su coche en el aparcamiento de un hotel de Phoenix en 1976 cuando iba a encontrarse con una fuente que le había prometido entregar las pruebas que incriminaban a políticos y hombres de negocio en fraudes inmobiliarios en el estado.

Aquel asesinato, todavía hoy sin resolver en parte, coincidió en el tiempo con el primer congreso nacional que el IRE celebraba en Indianápolis. No había duda de que Bolles había muerto a causa de las revelaciones periodísticas que había escrito, o de la historia en la que estaba trabajando en aquel momento, así que los miembros del recién nacido IRE comenzaron a discutir la mejor manera de responder periodísticamente al atentado. La violencia contra los periodistas estadounidenses no era nueva, pero en 1976 era extremadamente rara (incluso más que hoy día), y los periodistas reunidos en India- 
nápolis eran conscientes de que el asesinato de Bolles podría suponer, además de un ataque a la libertad de prensa, un precedente grave para otros periodistas investigadores si no se daba una respuesta profesional.

La respuesta fue un esfuerzo conjunto de una serie de periodistas de todo el país organizados en un equipo por el IRE que desentrañaron la corrupción entre políticos y empresarios en Arizona, terminando en esencia el trabajo que Bolles había dejado inacabado.

Financiado a través de donaciones, el equipo estaba dirigido por el director de Newsday y experto periodista de investigación, Robert Greene, quien lideraba un extenso grupo de periodistas y editores de diferentes lugares de los EE.UU. Después de tres meses de trabajo, el equipo produjo casi 80.000 palabras de texto detallando la corrupción en el estado de Arizona.

El 17 de marzo del 77 salía a la calle la primera entrega de la serie. En total treinta y siete editores y reporteros representando a 28 diarios y emisoras de TV habían trabajado en el proyecto. Se produjeron un total de 23 reportajes exponiendo la estructura del crimen organizado, la tradición del fraude inmobiliario, tráfico de drogas, juego, prostitución, la administración de justicia y las relaciones entre los políticos y la mafia en Arizona. La conclusión general del trabajo era que el estado de Arizona estaba seriamente minado por la corrupción, que existía una flagrante tolerancia entre las estructuras de poder y el estamento judicial para subvertir las leyes. La investigación revelaba además que las fuerzas de seguridad no disponían de medios para combatir la situación, sufrían falta de personal y de presupuestos, además de padecer un conflicto de jurisdicciones. Los resultados del trabajo periodístico se distribuyeron a 26 cabeceras de prensa (con la curiosa excepción del Arizona Republic, que se negó a publicar las investigaciones surgidas a raíz del asesinato de uno de sus periodistas), así como a 3 emisoras de TV y a través de la Associated Press a lo largo de todo el país. En la misma tradición de los muckrakers de principios de siglo, el equipo del IRE presentaba las pruebas no como abogados construyendo un argumento legal sino como periodistas queriendo contar una historia de corrupción en la vida pública ${ }^{11}$.

Nunca antes periodistas de diferentes medios habían trabajado juntos en una situación sin competencia para desarrollar una única historia. El IRE había hecho nacer un nuevo concepto en el Periodismo de Investigación: la cooperación directa entre periodistas de diferentes medios. En un artículo publicado en marzo del 77 como adelanto de las series del Proyecto Arizona, Tom Collins, en Newsday, calificaba el proyecto como "un inusual experimento de periodismo colectivo".

La publicidad que supuso el Proyecto catapultó al IRE a escala nacional. Greene, el director del proyecto y posteriormente presidente del IRE comentó que el Proyecto supuso el reconocimiento que el

${ }^{11}$ Los datos y comentarios del Proyecto Arizona, así como un detalle en profundidad de su desarrollo, puede consultarse en Aucoin (1997:33 y ss.), así como en el web conmemorativo realizado por el IRE al cumplirse el 30 aniversario del Proyecto (www.ire.org/history/arizona.html. última visita 19 de septiembre de 2005) 
IRE necesitaba como organización nacional. Hasta entonces la organización estaba asociada básicamente a los periodistas de la zona de Indianápolis que la habían hecho nacer, pero después del Proyecto Arizona el IRE obtuvo la atención nacional además de dos prestigiosos premios.

Un año después de la publicación del Proyecto Arizona, el Behavior Research Center, un servicio de sondeos de opinión pública con base en Phoenix, encuestó a un millar de cabezas de familia en la zona de las Montañas Rocosas y reveló que las series del IRE habían tenido un fuerte impacto a lo largo de Arizona y los estados limítrofes. El conocimiento de la serie de investigación alcanzaba el $80 \%$ entre los residentes en Arizona y el 64\% entre el resto de los residentes en la región de las Rocosas. (Aucoin, 1997 b:59-60)

Las series fueron seleccionadas como candidatas al Pulitzer en 1978 y premiadas con la Consciencein-Media Gold Medal de la American Society of Journalist and Authors, además de recibir el Premio nacional de la Ball State University.

Varios comentaristas sugirieron que el proyecto quedaría archivado en los anales de la historia del periodismo para ser estudiado en las facultades, emulado por los periodistas de investigación futuros y analizado por los historiadores. Entrado el siglo XXI, y como también señala Aucoin (Ibíd.) ninguna de esas predicciones se ha cumplido. Las menciones al mismo en los trabajos académicos raramente le dedican apenas una nota a pie de página.

A los quince meses de la conclusión del Proyecto, el IRE había sido adoptado en su seno por la facultad de periodismo más antigua de los EE.UU., la de Missouri-Columbia, y se legitimaba así mismo ante los ojos de la profesión periodística, lo que dejaba a la institución en buena disposición para afrontar los años 80, en los que el Periodismo de Investigación maduraría hacia una corriente de actividad habitual en el periodismo norteamericano.

Los embriagadores años del Watergate se desvanecieron en los 80, pero la memoria de los periodistas denunciando corrupciones de magnitud nacional gracias a su persistente búsqueda de información se estableció como un ideal para la profesión. Los periodistas, incluso aquellos que no investigaban por norma general, comenzaban a hacerlo. El descubrimiento de irregularidades había conseguido glamour y el status del periodismo de calidad.

Desde los primeros años 80 el periodista que exponía lo venal y lo corrupto se convertía pronto en poco menos que una celebridad, el típico héroe americano. Woodward y Bernstein eran la referencia. La televisión y el cine apuntalaban el glamour de este tipo de periodismo y "60 minutes" se convertía por entonces en el programa más popular de la televisión. En el 82, según una encuesta de Gallup, cuatro de cada cinco norteamericanos defendían el Periodismo de Investigación y dos de cada tres reclamaban que los medios hiciesen todavía más investigaciones (Ibíd.). Esta mezcla de reverencia y 
popularidad hizo subir todavía más al IRE, lo puso en posición de influenciar en la evolución de la especialidad y le dio seguridad financiera.

En 1985 casi todos los diarios y televisiones practicaban investigaciones de forma regular u ocasional, pero solo un 20 por ciento de los medios tenían más de un profesional dedicado en exclusiva a la especialidad, y la mayoría no tenía ninguno, reseña Aucoin (Ibíd.). La afiliación al IRE crecía solo por el atractivo que suponía para los periodistas que reconocían la importancia de la especialidad y querían fundir las técnicas de investigación con la rutina del periodismo diario, o que buscaban poder encontrar tiempo para trabajos de investigación.

Los premios anuales establecidos por el IRE sirvieron de foro para continuar la discusión sobre la definición del Periodismo de Investigación. De hecho, era el único foro dentro de la organización donde se había dado una definición de la especialidad y los criterios para otorgar los galardones se convirtieron en la guía para tal definición. Cuando se estableció por primera vez el programa de premios, el IRE adoptó la definición dada por Bob Greene:

Se trata de un trabajo que es sustancialmente el producto de la propia iniciativa del periodista, que se esfuerza en descubrir hechos o datos que alguien trata de guardar en secreto; debe tratarse de asuntos de importancia para la audiencia del medio; la información debe ser exacta y bien documentada, además de bien escrita y propiamente presentada, con un seguimiento post-publicación incluido.

Un punto de conflicto en la discusión de cara a una definición generalmente aceptada era el requerimiento de que el Periodismo de Investigación debía descubrir secretos. Era un tema ya debatido en los 70 y que continuaría durante los 80 . En el 74, durante un discurso ante la comunidad de editores, Katherine Graham, editora del Washington Post, argumentó que el Periodismo de Investigación incluía, a su juicio, tanto aquellas historias que revelaban secretos e irregularidades como historias que buceaban en el sistema y en las instituciones, en lo público y en lo privado, para averiguar cómo funciona realmente, quién ejerce el poder, a quién beneficia y a quién daña. El Post había publicado un estudio detallado del servicio postal norteamericano en aquel 1974 en el que revelaba ineficiencias y mala gestión, aunque no corrupción. Diez años después un miembro del jurado de los premios del IRE, el profesor de la Universidad de Missouri, George Pica, se opuso a la visión de la especialidad del IRE dada la limitación que suponía el aspecto de la revelación de secretos. En un memorando al consejo directivo, Pica explicaba que las series de Donnald Barlett y James Steele sobre la gestión de residuos nucleares publicadas en el Philadelphia Inquirer no encajaba con la definición de la especialidad del IRE: no revelaba secretos, pero era, sin embargo, Periodismo de Investigación. En respuesta a Pica y a otros como él que no compartían la exigencia sobre el secreto en la especialidad, el consejo directivo del IRE alteró los criterios para juzgar los candidatos a sus premios, dejando claro que la revelación de eventos y datos secretos sería una consideración y no un requerimiento en el futuro. Además, se modificaba el requerimiento de que el trabajo fuese realizado a iniciativa personal del periodista, en el sentido de que 
debía ser, sustancialmente, producto de la actividad del periodista ${ }^{12}$. Cuando en 1986 el IRE y la facultad de periodismo de la Universidad de Missouri sondearon la opinión de los periodistas sobre este particular, estos afirmaron estar de acuerdo en su mayoría con la definición adoptada.

La reputación de la especialidad en los EE.UU. y de su estandarte organizacional se consolidaría definitivamente en la segunda mitad de la década de los 80. El 4 de julio de 1987, Editor and Publisher editorializaba en defensa del IRE por introducir al Periodismo de Investigación en la corriente principal del periodismo estadounidense, una clara evidencia y reconocimiento de lo que la organización había conseguido en su primera década de existencia. Más aún, Editor and Publisher reconocía que el trabajo del IRE había elevado la calidad del periodismo en general. Un año antes, la misma publicación había editorializado que el periodismo en papel estaba mejorando y que el Periodismo de Investigación era el motor de esa mejora, el referente de lo que el periodismo debería ser.

Pero de los años ochenta también deben ser destacados otros factores como la aparición y adopción del Periodismo Asistido por Ordenador (PAO, traducción del anglosajón CAR: Computer-Assisted Reporting), los recortes presupuestarios en las empresas periodísticas en la segunda mitad de la década, que dieron al traste con el entusiasmo de los editores respecto a una especialidad como el Periodismo de Investigación, que pese a la calidad, suponía grandes inversiones; la emergencia de las investigaciones periodísticas editadas en formato de libro, que se convertían casi automáticamente en éxitos de venta editorial; y una serie de sonados procesos judiciales derivados de la publicación de investigaciones que hicieron a la especialidad percatarse de la necesidad de mirarse en el espejo y revisar sus técnicas y valores.

Si el Watergate y el Proyecto Arizona supusieron las cimas de la especialidad en la década de los setenta, fue el trabajo colectivo del Philadelphia Inquirer lo que ejemplificaba las posibilidades del Periodismo de Investigación en los ochenta. No fue ninguna investigación aislada del diario sino el compromiso general y la dedicación del diario bajo la dirección de Eugene Roberts, lo que le convirtió en el estandarte de la calidad en los ochenta. Roberts mejoró la cobertura informativa del diario con el suministro de recursos técnicos y económicos para producir largas y profundas investigaciones. Bajo la dirección de Roberts El Inquirer obtuvo diecisiete premios Pulitzer entre 1985 y 1990, muchos de ellos en el apartado de investigación. Para Roberts, tal como afirmó en 1988 durante una serie de conferencias en la University of Southern California, "el mejor periodismo -sea corto o largo- es siempre de investigación, el que busca, busca y busca... En el Inquirer, Periodismo de Investigación significa liberar a un redactor de las restricciones de tiempo y espacio y dejar que su artículo informe realmente al público sobre una situación de vital importancia. Significa entender cómo la sociedad ha crecido tan rápido y tan complejamente como para no ser ya puesta al corriente, tan solo, con meras informaciones y fotos

\footnotetext{
${ }^{12}$ Recogido en los Archivos del IRE. IRE Resource Center.
} 
en color. Algunos periódicos les fallan a sus lectores al rechazar hacer cualquier tipo de investigación periodística", sentenciaba ${ }^{13}$.

Pero las instituciones que soportan al Periodismo de Investigación -diarios, revistas, editores literarios, etc.- siempre están sujetas a la presión de sus departamentos financieros. A finales de los 80 y principios de los 90, la crisis económica sacudió a la industria del periodismo al tiempo que se incrementaba la concentración de los medios, lo que implicaba una mayor presión sobre los beneficios. Durante la convención nacional del IRE de 1990 el editor ejecutivo del Wall Street Journal, Normal Pearlstine, advertía que el Periodismo de Investigación afrontaba su período más difícil dado que los márgenes operativos decrecían y la presión sobre los editores y los ejecutivos de redacción crecía a diario. Pearlstine predecía que dicha presión iba a ser más grande que cualquier fenómeno visto en el sector hasta el momento. Mientras el interés en la especialidad crecía entre los periodistas, sus jefes restringían su práctica para ahorrar dinero.

Convertido ya de forma definitiva en parte de la corriente principal del periodismo norteamericano, el Periodismo de Investigación seguía, sin embargo, siendo apoyado por muchos medios a pesar de los problemas económicos. Muchos ejecutivos de prensa apoyaron la idea de Lawrence K. Beaupre, director ejecutivo de la cadena Westchester Rockland Newspapers, quien afirmó en dicha convención que los proyectos de investigación podían volverse más modestos, pero que el Periodismo de Investigación se había convertido en algo demasiado importante en la labor de los diarios como para que desapareciese. Los recortes presupuestarios afectaron a la especialidad, pero en la mayoría de los casos solo hicieron que los editores se volviesen más selectivos en los proyectos a desarrollar en lugar de eliminarlos de las redacciones. De hecho, una faceta del Periodismo de Investigación vivió un avance considerable en los 80: el uso de análisis informáticos para producir historias de investigación y reportajes en profundidad. El PAO maduraba en aquellos 80, concebido muy a menudo como una herramienta técnica al servicio de la puesta al día en recursos tecnológicos del tradicional Periodismo de Investigación.

Durante los 90, la especialidad era ya un fenómeno nacional en EE.UU. Las emisoras de televisión por cable y las cadenas públicas emitían de forma estable programas de investigación, desde el sensacionalista y éticamente reprobable "A Current Affair" de la NBC, hasta ejercicios periodísticos mucho más serios como el "20/20" de la ABC. Además, Internet y el World Wide Web comenzaba a hacer posible el acceso de millones de personas de todo occidente a las investigaciones realizadas por todo tipo de medios, locales y nacionales, estadounidenses. Por primera vez desde la época dorada de los muckrakers, la publicación de problemas locales tenía potenciales audiencias nacionales y la explicación de los problemas nacionales tenía potenciales audiencias masivas. El Periodismo de Investigación en los 90, al igual que en los primeros años del siglo xx, se convertía en una característica definitoria del

\footnotetext{
${ }^{13}$ Citado en Aucoin, 1997b
} 
periodismo norteamericano: cuando se hacía bien sentaba las bases para definir la calidad de un medio, pero cuando se hacía mal era la vía de entrada de todo tipo de quejas contra el oficio.

Con la consolidación llegaban las críticas y el terreno para las mismas se abonó desde la aparición de una de las series ("América, What Went Wrong") del conjunto de trabajos antes comentado del Philadelphia Inquirer, y se agudizaron al máximo con investigaciones de gran calado como "Dark Alliance: The Story Behind the Crack Explosion”, del San José Mercury News o el escándalo destapado por la ABC sobre el uso de productos químicos para conservar alimentos en la cadena de supermercados Food Lion, que eran vendidos como frescos. Se trataba de casos en que los periodistas abordaban temas de gran dificultad usando, en algunos casos, técnicas popularmente polémicas pero de acreditada reputación en la deontología de las ciencias sociales (cuando se utilizan dentro de unos límites procedimentales y justificación de contexto) como la utilización de cámaras ocultas o fuentes no del todo identificadas para destapar escándalos de otra forma imposibles de conseguir periodísticamente. El profesor de ética periodística de la Missouri School of Journalism, Edmund Lambert, argumentó posteriormente en su libro Committed Journalism (1992) que se necesitaba un Periodismo de Investigación aún más sofisticado para afrontar el desafío de examinar a un mundo cada vez más y más complejo, poniendo en el terreno de la informática -del PAO- la pelota de la sofisticación y la aportación de evidencias.

En efecto, la utilización de las hojas de cálculo y las bases de datos, además de algún otro tipo de programa informático para la recopilación y procesamiento de grandes conjuntos alfanuméricos pasó a convertirse en el recurso técnico más apreciado de los periodistas de investigación más innovadores; hasta el punto de que, al menos una parte de los nuevos expertos llegó a considerar que el Periodismo de Investigación contemporáneo ya solo tendría mérito si era capaz de utilizar estas nuevas herramientas en la planificación y desarrollo de su trabajo. De ello ya hace tres lustros.

\section{Referencias bilbiográficas}

- Anderson, J. (1973): The Anderson Papers. New York. Random House.

- Aucoin, J.L. (1993): IRE and the Evolution of Modern American Investigative Journalism, 1960-1990.

- Aucoin, J.L. (1995a): “The Early Years of IRE: The Evolution of Modern Investigative Journalism”, American Journalism, vol. 12:4, pp. 425-443.

- Aucoin, J.L. (1995b): “The Re-emergence of American Investigative Journalism 1960-1975”, Journalism History, vol. 21, pp. 3-15.

- Aucoin, J.L. (1997a): "Historiographic Essay: The Investigative Tradition in American Journalism”, American Journalism, vol. 14:3-4, pp. 317-329.

- Aucoin, J.L. (1997b): Investigative Reporters and Editors, the Arizona Project, and the Evolution of American Investigative Journalism. Evergreen, Alabama. Raging Cajun Books. 
- Behrens, J.C. (1977): The Typewriter Guerrillas: Closeups of 20 Top Investigative Reporters. Chicago. Nelson-Hall.

- Benjaminson, P. /Anderson, D. (1990): Investigative Reporting. Ames. Iowa State University Press.

- Boylan, J. (1986): 'Declarations of Independence: a Historian Relects on an Era in which Reporters Rose Up to Challenge -and Change- the Rules of the Game', Columbia Journalism Review, noviembrediciembre. Pp. 29-45.

- Buschel, B. /Robbins, A. et al. (1973): The Watergate File. New York. Flash Books.

- Casal, F. (2005): El Periodismo de Investigación Asistido Por Ordenador y de Precisión aplicado a la prensa local y regional: el contraste entre Estados Unidos y un modelo de futuro para Galicia. Tesis Doctoral. Universidad Complutense de Madrid.

- Christianson, K.S. (1972): ‘The New Muckraking', The Quill, Julio. p. 12.

- Dader, J.L. (1997): "Principios y limitaciones esenciales del periodismo de investigación a lo largo de su historia", en Dader, J. L.: Periodismo de Precisión. La Vía socioinformática de descubrir noticias. Madrid. Síntesis, pp. 28-41.

- Dygert, J.H. (1976): The Investigative Journalist: Folk Heroes of a New Era. Englewood Cliffs, N.J. Prentice-Hall.

- Editor \& Publisher (1975): 'Investigative Reporters Form Own Service Association', Editor \& Publisher, 08-III-1975.

- Eszterhas, J. (1975): 'The Toughest Reporter in America', Rolling Stone, 10 y 24 de abril.

- Francke, W.T. (Team Investigation in the 19t Century: Sunday Sacrifices by the Reporting Corps.', Presentación inédita en la convención anual de la AEJMC 1988.

- Freivogel, B. (1973): 'Public Affairs Reporting Assessed at Press Meeting', Editor \& Publisher, vol.106, p. 7.

- Investigative reporters and editors. (1977): The Arizona Project: Reprint of a 1977 series. Columbia, Mo. IRE.

- Kirtz, B. (1975): 'Investigative Reporters Relate How They Operate', Editor \& Publisher, vol.108, p. 24.

- Kroeger, B. (1994): Nellie Bly: Daredevil, Reporter, Feminist. New York. Times Books.

- Lambert, E. (1992): Committed Journalism. Bloomington, In. Indiana University Press.

- Macintyre, A.C. (1981): After Virtue: A Study in Moral Theory. Notre Dame, Ind. University of Notre Dame Press. 
- McWilliams, C. (1973): “The Continuing Tradition of Reform Journalism”, en Harrison, J. M. (ed.): Muckraking: Past, Present, and Future. University Park, Pa. Pennsylvania State University Press, p. 129.

- Meyer, P. (1979): Precision Journalism: A Reporter's Introduction to Social Science Methods. Bloomington. Indiana University Press.

- Miraldi, R. (1990): Muckraking and Objectivity: Journalism's Colliding Traditions. New York. Greenwood Press.

- Mollenhoff, C.R. (1981): Investigative Reporting: From Courthouse to White House. New York, London. Macmillan; Collier Macmillan.

- Protess, D., et.al. (1991): The Journalism of Outrage: Investigative Reporting and Agenda Building in America. New York. Guilford Press.

- Senia, A. (1978): “The Arizona Project... A Year Later”, The Quill, Julio-agosto. pp. 10-28.

- Serrin, J. / Serrin, W. (2002): Muckraking!: the Journalism that Changed America. New York. New Press.

- Stein, H. (1979): “American Muckrakers and Muckraking: the 50-years Scholarship”, Journalism Quarterly, vol. 56:1, pp. 9-17.

- Watson, C. (1973): “Size Termed Unessential for Exposes”, Editor \& Publisher, vol. 101, p. 48.

- Weinberg, S. (1992): Telling the Untold Story: How Investigative Reporters are Changing the Craft of Biography. Columbia. University of Missouri Press.

- Wendland, M.F. (1988): The Arizona Project. What a Team of Investigative Reporters Discovered About Crime and Corruption in Today's Wild West. Mesa. Blue Sky.

- Williams, P.N. (1978): Investigative reporting and Editing. Englewood Cliffs, N.J. Prentice-Hall. 
